DOI: 10.1002/adma.((please add manuscript number))

\title{
Mechanically Tunable, Self-Adjuvanting Nanoengineered Polypeptide Particles
}

By Jiwei Cui, Robert De Rose, James P. Best, Angus P. R. Johnston, Sheilajen Alcantara, Kang Liang, Georgina K. Such, Stephen J. Kent,* and Frank Caruso*

[*] Dr. J. Cui, ${ }^{[+]}$J. P. Best, Dr. A. P. R. Johnston, K. Liang, Dr. G. K. Such, Prof. F. Caruso Department of Chemical and Biomolecular Engineering

The University of Melbourne

Parkville, Victoria 3010, Australia

E-mail: fcaruso@unimelb.edu.au

Dr. R. De Rose, ${ }^{[+]}$S. Alcantara, Prof. S. J. Kent

Department of Microbiology and Immunology

The University of Melbourne

Parkville, Victoria 3010, Australia

Email: skent@unimelb.edu.au

[+] J. Cui and R. De Rose contributed equally to this work.

Keywords: vaccine delivery, nanotechnology, mesoporous silica, polypeptide particles, CpG, adjuvant

Vaccines are the most cost effective public health measure for infectious diseases, since they provide long-term immunity against a given pathogen. ${ }^{[1]}$ A principal component of many vaccines is an adjuvant, which enhances the immunogenicity of an antigen. Adjuvants act by activating dendritic cells (DCs) or other innate immune cells, or by enhancing antigen uptake and presentation by DCs. ${ }^{[2]}$ Currently, few adjuvants are licensed for use in human vaccines. Non-targeted delivery of adjuvanted vaccines can result in widespread immune-activation and have been linked to adverse local and systemic reactions. New candidate adjuvants are being evaluated in combination with systems to effectively deliver adjuvant/antigen combinations. $^{[3]}$ In recent years, the study of the innate immune system has revealed that numerous immunostimulatory molecules on viruses, bacteria and parasites alert the host to an infection and act as natural adjuvants that are essential in initiation of an immune response. These ligands are referred to as Pathogen Associated Molecular Patterns (PAMPs) and the host cell receptors that identify them as Pattern Recognition Receptors (PRRs). Such 


\section{ADVANCED}

molecules are well suited for vaccine development and can be readily included in particulate vaccine carriers to promote an immune response to the encapsulated antigen. Particulate vaccine carriers can thus deliver the antigenic payload and the adjuvant to the same antigen presenting cell and more effectively initiate an immune response. An additional benefit is the capacity to deliver lower doses of vaccine to the target cells (DCs) and simultaneously reduce unwanted side-effects. Developments of vaccines are also challenged by the nature of the components, such as nucleic acids or peptides, which are often sensitive to degradation by host enzymes prior to reaching the target cell. ${ }^{[4]}$ Thus, the incorporation of antigens and adjuvants into nanoengineered carriers has the potential to dramatically improve treatment options by protecting the cargo and targeting to the relevant immune cell to initiate an immune response. ${ }^{[5]}$

Polymer carriers have received increasing interest for vaccine delivery, due to the flexibility of their compositions and structures, as well as their finely controlled and responsive properties. ${ }^{[6]}$ Antigens and adjuvants have been encapsulated in layer-by-layer (LbL) polymer capsules for vaccine delivery. ${ }^{[7]}$ We have previously shown that poly(methacrylic) acid capsules, cross-linked with disulfide bonds and loaded with ovalbumin or immunogenic peptides, can illicit an immune response in vitro ${ }^{[7 \mathrm{a}, 7 \mathrm{~b}]}$ and in vivo. ${ }^{[7]}$ De Geest and coworkers have shown that antigen-loaded dextran sulfate/poly-Larginine capsules offer viral and tumor immunity in vitro and in vivo. ${ }^{[7 \mathrm{~d}]}$ However, the LbL preparation method can be time-consuming and generally requires multiple polymer adsorption steps. Polymer replica particles, obtained by templating mesoporous silica (MS) particles (which have high surface areas and pore sizes in the range of $2-50 \mathrm{~nm}^{[8]}$ ), represent alternative carriers that offer promise for use as vaccine delivery vectors. ${ }^{[9]}$ This templating approach has been shown to be versatile in terms of polymer type and cross-linking strategy, and also can be fine-tuned to tailor the composition, morphology and porosity of the 
Submitted to

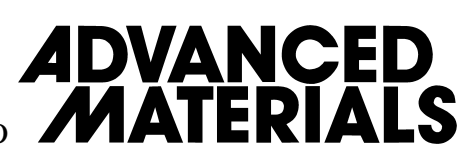

nanostructures, and their responsiveness to biological stimuli. ${ }^{[9]}$ Different therapeutics (i.e., doxorubicin, paclitaxel, thiocoraline, and a brain-derived neurotrophin factor) and plasmid DNA/peptide hormones have been loaded into such polymer replica particles for drug delivery ${ }^{[10]}$ and transfection, ${ }^{[11]}$ respectively. However, for effective vaccine delivery there is a need to develop particles loaded with adjuvants to prime for potent and enduring immunity.

In this work, redox-responsive polypeptide replica particles with controlled loading capacity were prepared via MS-templated assembly for the delivery of an oligonucleotide adjuvant, CpG, with a sequence of 5'-GGG GGA CGA TCG TCG GGG GG-3'. CG dinucleotides, in particular sequence contexts, on viruses, bacteria and parasites are typically unmethylated and thus differ from mammalian CG, which display more frequent methylation of the cytosine nucleotide. This difference is recognized by the PRR known as Toll-like receptor TLR9, which is found on a subset of dendritic cells (DC) known as plasmacytoid DCs (pDC). Previous work on LbL capsules loaded with both antigen (ovalbumin) and adjuvant $(\mathrm{CpG})$ were recently shown to have an improved immune response compared to capsules that only contained antigen. ${ }^{[7]}$ To achieve the encapsulation of CpG in our polymer replica particles, CpG was conjugated onto poly(L-glutamic acid) (PGA) to form PGA-CpG conjugates. The CpG-loaded polymer particles were obtained through infiltration of the PGACpG conjugates into MS particles, followed by cross-linking of the PGA chains, and subsequent removal of the porous silica templates (Scheme 1a). This approach offers a number of distinct advantages. First, DNA was covalently conjugated to a polypeptide with a disulfide bond to prepare polymer conjugate particles, which provides a new approach to assemble redox-responsive oligonucleotide-loaded particles. Second, a versatile cross-linking approach exploiting thiol-disulfide exchange was used to create disulfide-stabilized polymer hydrogel carriers. This was achieved using a second PGA conjugate with thiol functionality. In this way, the polymer served as a cross-linker to generate single-component polymer 


\section{Submitted to \\ ADVANCED}

particles. Third, the elasticity and the cargo loading capacity of the obtained particles can be tuned by varying the amount of cross-linker used to stabilize the polymer particles. The resultant particles demonstrated successful delivery of their cargo and subsequent activation of primary human blood pDCs (Scheme 1b). In vitro experiments showed that the level of pDCs activation could be controlled by simply tuning the loading capacity of the polymer particles. To our knowledge, this study represents the first example of the preparation of vaccine particles with mechanical properties that are tunable by controlling the cross-linking density, which also governs the cargo loading capacity and cellular activation.

To construct polymer particles with a high loading capacity, MS particles with a bimodal pore structure (smaller mesopores in the 2-3 nm range and larger mesopores between 10-40 nm) were used as templates, which were synthesized according to a modified literature method (See Supporting Information). ${ }^{[12]}$ Nitrogen sorption data indicated that the MS particles had a surface area of $893 \mathrm{~m}^{2} \mathrm{~g}^{-1}$ and a pore volume of $1.34 \mathrm{~cm}^{3} \mathrm{~g}^{-1}$. Transmission electron microscopy (TEM) and scanning electron microscopy (SEM) revealed that the average size of the MS particles was $800 \mathrm{~nm}$ (Supporting Information, Figure S1). These MS particles with large mesopores and extensive surface area have proven to be excellent templates for the assembly of polymer particles from large molecular weight macromolecules for biological applications. ${ }^{[10 a]}$

For the encapsulation of $\mathrm{CpG}$, thiolated $\mathrm{CpG}$ was conjugated on pyridine dithioethylamine (PDA)-functionalized PGA (PGA ${ }_{\mathrm{PDA}}, 12 \%$ modification) via thiol-disulfide exchange to form PGA-CpG conjugates, where thiolated CpG reacted with a pyridyldithio side chain of the PGA $A_{P D A}$ to release a corresponding 2-mercaptopyridine molecule (Supporting Information, Figure S2a). ${ }^{[13]}$ The PGA-CpG conjugates were infiltrated into amine-functionalized MS particles and cross-linked with thiolated PGA (PGA SH $_{10}$ 10\% modification) (Supporting Information, Figure S2b). The PGA $\mathrm{SH}_{\mathrm{SH}}$ concentration was systematically varied to tune the 
degree of cross-linking in the particles. Following removal of the MS template, PGA-CpG particles were obtained. To visulize the encapsulation of the oligonucleotides, FITC-labeled CpG was used to prepare the particles. The PGA-CpG particles cross-linked with PGA $\mathrm{SH}_{\mathrm{SH}}$ were well dispersed in PBS buffer with a diameter of $\sim 1.3 \mu \mathrm{m}$ (Figure 1a), which represents approximately $60 \%$ swelling from the original template size. This swellability can be attributed to the electrostatic repulsion between the glutamic acid groups ( $\left.\mathrm{p} K_{\mathrm{a}} \sim 4.3\right) .{ }^{[14]}$ Green fluorescence indicated that CpG had been encapsulated in the PGA particles. Interestingly, the fluorescence intensity of the PGA-CpG particles increased when a higher concentration of PGA $_{S H}$ was used, as observed in Figure 1a (images a3 and a4). TEM images showed that the contrast of the particles also increased with linker concentration (Figure 1b). It is likely that highly cross-linked particles resulted in higher loading of CpG. To investigate this, we quantified the amount of CpG per particle cross-linked with different amounts of PGA $\mathrm{SH}_{\mathrm{SH}}$ by flow cytometry and fluorescence spectrophotometry. The concentration of PGA-CpG particles was determined by flow cytometry. These particles were then exposed to a dithiothreitol (DTT) solution (pH 8), which resulted in the disassembly of the particles and release of FITC-labeled CpG. Using a fluorescence calibration curve for FITC-labeled CpG, we deduced the amount of CpG encapsulated in each particle. The amount of CpG per particle as a function of linker concentration is shown in Figure 2a and demonstrates that the loading capacity of the particles increases with increasing cross-linking degree.

Since the degree of cross-linking altered the loading capacity, it was expected that the particles would have different mechanical properties, which has been shown to affect cell association. $^{[15]}$ Here, the mechanical characterization of the PGA-CpG particles was performed using atomic force microscopy (AFM). Consistent with the CpG loading capacity, the elastic Young's modulus $\left(E_{Y}\right)$ of the PGA-CpG particles increased with increasing crosslinker concentration (Figure 2a). However, it reached a plateau of $25 \mathrm{kPa}$ as the cross-linker 


\section{ADVANCED}

Submitted to ACASTS

loading capacity was approached. We postulate that increasing the cross-linker concentration enhances the cross-linker loading amount and the degree of cross-linking, resulting in an increase in particle $E_{Y}$. When the loading amount of the cross-linker becomes saturated, the cross-linking density and $E_{Y}$ plateau. PGA-control particles were prepared using the same approach but without the immunostimulatory properties of CpG. These particles were loaded with oligonucleotide of the same length and nucleotide composition, but the CG nucleotides

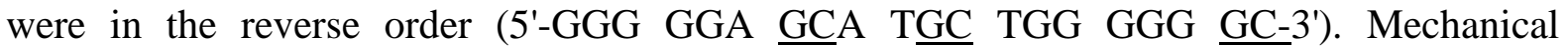
measurements indicated that the Young's modulus of the PGA-Control and PGA-CpG particles cross-linked with same amount of PGA $\mathrm{SH}_{\mathrm{SH}}$ was quite similar (Figure 2b), which is seven times lower than that of PGA particles without DNA (data not shown).

To assess the association and immunostimulatory capacity of these particles, PGA-CpG and PGA-control particles were cultured with human peripheral blood mononuclear cells (PBMC) at $37^{\circ} \mathrm{C}$ for $9.5 \mathrm{~h}$. The association of DCs with both particles was quantified using flow cytometry. The percentage of pDC association with either PGA-CpG or PGA-control particles increased as the cross-linking increased from 0.05 to $0.2 \mathrm{mg} \mathrm{mL}^{-1}$ and then plateaued from 0.2 to $2 \mathrm{mg} \mathrm{mL}^{-1}$ (Figure 3a). This association percentage is much higher than that of PGA particles without DNA (Supporting Information, Figure S3). The cell association increased with increasing cross-linker concentration from 0.05 to $1 \mathrm{mg} \mathrm{mL}^{-1}$ and then decreased at a cross-linker concentration of $2 \mathrm{mg} \mathrm{mL}^{-1}$. We have previously observed that the presence of functionalities such as thiols on the particle surface play a dominant role in cell association, ${ }^{[16]}$ and it is likely that the presence of DNA has a similar effect. The level of association to pDC was similar for PGA particles loaded with either the CpG or control sequence. Myeloid DC (mDCs) also showed similar levels of association to PGA-CpG and PGA-control particles (Supporting Information, Figure S4a). This may be due to the additional benefit of surface DNA distribution. Increasing the degree of cross-linking and 
ed activation of the pDC population for the PGA-CpG particles only, as evidenced by upregulation of CD40 and CD83 on the cell surface (Figure 3b). However, there was no activation for the equivalent amount of free CpG. Activation by CpG was specific for the pDC subset as particles with the control sequence exhibited lower levels of activation. In addition, the proportion of CD83+/CD40+ mDC was similar for both types of PGA-DNA particles and not elevated above background levels of activation, as expected, since the receptor for $\mathrm{CpG}$ is not expressed in mDCs (Supporting Information, Figure S4b).

In conclusion, we have reported the preparation of DNA-loaded polypeptide particles via mesoporous silica templating and a versatile cross-linking approach of thiol-disulfide exchange for the delivery of adjuvants. The cargo loading capacity was tuned by the amount of cross-linker used to stabilize the polymer particles, and it was found that DNAfunctionalized particles exhibited higher binding to pDCs than unfunctionalized particles. The resultant polypeptide particles were successfully used to deliver their cargo to primary human pDCs and activate pDCs. In vitro experiments showed that the polypeptide particles with higher loading capacity resulted in increasing activation of pDCs. This should lead to substantially improved vaccine immunogenicity. The MS templating method and tunable cross-linking technique could be extended to other systems to provide hydrogel carriers with tailored properties for a range of therapeutic delivery applications.

\section{Experimental Section}

AFM Force Spectroscopy Measurements and Analysis: The mechanical characterization of the PGA-CpG particles was performed using a Nanowizard II AFM (JPK Instruments AG, Berlin, Germany), loaded with high-reflectance cantilevers with nominal spring constants ranging from 0.03 to $0.09 \mathrm{~N} \mathrm{~m}^{-1}$ (CSC38/Cr-Au, MikroMasch, Estonia). Prior to measurement, cantilevers and glass substrates were immersed in a 30 vol\% isopropanol 
Submitted to

\section{ADNANED
MAATRRALIS}

solution, followed by Milli-Q water, and then further cleaned using oxygen plasma for $180 \mathrm{~s}$ (Harrick Plasma, 0.1 L min ${ }^{-1}$, 29.6 W, 300 mTorr). Cleaned glass substrates were treated with a monolayer of poly(ethyleneimine) (PEI, $1 \mathrm{wt} \%$ ) to promote particle immobilization. Cantilevers were calibrated in air on the PEI-coated substrates using the thermal noise method, as described in literature, prior to measurement. ${ }^{[17]}$ Then, $1 \mu \mathrm{L}$ of the particle suspension was dispensed onto the substrate and ca. $150 \mu \mathrm{L}$ PBS was added. The cantilever was then fully immersed in the droplet, and the inverse optical lever sensitivity (InvOLS) of the cantilever was re-measured in buffer. Particles immobilized on the surface could be visualized optically in situ using a Leica DMI 4000B optical microscope and 40× objective lens (Leica Microsystems GmbH, Wetzlar, Germany). Force spectroscopy measurements were then made on the hydrogel particles by optically aligning the cantilever tip above individual particles, and initiating an approach-retract force cycle with a constant piezo velocity of $4.0 \mu \mathrm{m} \mathrm{s}^{-1}$. It was seen that when deforming immobilized particles, the forcedisplacement gradient differed significantly from the InvOLS of the PEI-coated substrate.

Collected force spectra were analyzed using accompanying JPK data processing software. A baseline was firstly subtracted from the non-contact z-range of the force-displacement data, a probe/surface contact point assigned, and the effect of cantilever bending subtracted to result in force-deformation $(F-\delta)$ data. The Young's modulus $\left(E_{Y}\right)$ was determined over the first $10 \%$ of total deformation, using the Hertz-Sneddon relationship for a conical indenter: $F=\frac{E_{Y}}{1-v^{2}} \frac{2 \tan \alpha}{\pi} \delta^{2}$

where $v$ is the Poisson ratio and $\alpha$ is the semi-opening angle of the conical indenter. ${ }^{[18]}$ The Poisson ratio was assumed to be 0.5 , a reasonable value for incompressible isotropic elastic materials, while $\alpha$ was determined as $15.2 \pm 0.4^{\circ}$ through SEM analysis of the cantilever batch. To achieve representative $E_{Y}$ values for the PGA hydrogel particles, at least 10 particles were analyzed for each sample. 
In Vitro Experiments: Peripheral blood mononuclear cells (PBMC) prepared from human donor blood were incubated with particles in RPMI media containing $10 \%$ fetal calf serum at a ratio of 100:1 (particles:PBMC) at $37^{\circ} \mathrm{C}$ for $9.5 \mathrm{~h}$ in a humidified atmosphere containing 5\% $\mathrm{CO}_{2}$. pDCs were identified as Lineage (Lin)1-/CD123+/HLA-DR+ cells. mDCs were Lin1-/HLA-DR+/CD11c+. Backgating to a FSC-A vs. SSC-A plot revealed pDCs with slightly lower granularity than mDCs. Activation of DCs was determined by CD83 and CD40 co-expression. CD83 is a maturation marker and CD40 is a marker of activation. PBMC cultured in media alone were used to determine background activation of pDCs ( 3\% for pDCs and $\sim 27 \%$ for mDCs). Particle association with cells was measured from the fluorescence imparted to the cells by Pacific blue-labeled PGA particles and the threshold for association was determined from PBMC cultured without particles. All antibodies were purchased from Becton Dickinson (San Jose, CA). Lineage 1 cocktail = CD3+14+16+19+20+56.

\section{Supporting Information}

Supporting Information is available from the Wiley Online Library or from the author.

\section{Acknowledgements}

This work was supported by the Australian Research Council under the Australian Laureate Fellowship (F.C., 120100030) and Discovery Project schemes (F.C., 130101846) and by the National Health and Medical Research Council (NHMRC) program (S.J.K., 510448) and fellowship awards (S.J.K., 508937). J.C. is the recipient of an Australian Research Council Super Science Fellowship (FS110200025). J.C. also acknowledges the support of the Early Career Research Grant from the University of Melbourne. R.D. is supported by the NHMRC Career Development Fellowship (APP1011578). Dehong Chen is thanked for the nitrogen sorption experiments. 
Submitted to

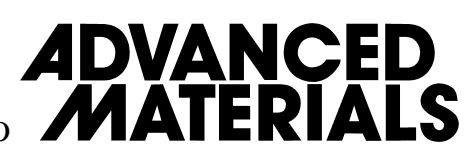

Received: ((will be filled in by the editorial staff))

Revised: ((will be filled in by the editorial staff)) Published online: ((will be filled in by the editorial staff))

[1] S. De Koker, B. N. Lambrecht, M. A. Willart, Y. van Kooyk, J. Grooten, C. Vervaet, J. P. Remon, B. G. De Geest, Chem. Soc. Rev. 2011, 40, 320.

[2] a) B. Guy, Nat. Rev. Microbiol. 2007, 5, 505; b) C. Rueckert, C. A. Guzmán, PLoS Pathog. 2012, 8, e1003001.

[3] A. L. S. John, C. Y. Chan, H. F. Staats, K. W. Leong, S. N. Abraham, Nat. Mater. 2012, 11, 250.

[4] D. N. Nguyen, J. J. Green, J. M. Chan, R. Langer, D. G. Anderson, Adv. Mater. 2009, $21,847$.

[5] A. P. R. Johnston, G. K. Such, S. L. Ng, F. Caruso, Curr. Opin. Colloid Interface Sci. 2011, 16, 171.

[6] a) M. A. C. Stuart, W. T. S. Huck, J. Genzer, M. Müller, C. Ober, M. Stamm, G. B. Sukhorukov, I. Szleifer, V. V. Tsukruk, M. Urban, Nat. Mater. 2010, 9, 101; b) M. Elsabahy, K. L. Wooley, Chem. Soc. Rev. 2012, 41, 2545; c) A. C. Rice-Ficht, A. M. Arenas-Gamboa, M. M. Kahl-McDonagh, T. A. Ficht, Curr. Opin. Microbiol. 2010, 13, 106.

[7] a) R. De Rose, A. N. Zelikin, A. P. R. Johnston, A. Sexton, S. F. Chong, C. Cortez, W. Mulholland, F. Caruso, S. J. Kent, Adv. Mater. 2008, 20, 4698. b) S.-F. Chong, A. Sexton, R. De Rose, S. J. Kent, A. N. Zelikin, F. Caruso, Biomaterials 2009, 30, 5178; c) A. Sexton, P. G. Whitney, S. F. Chong, A. N. Zelikin, A. P. R. Johnston, R. De Rose, A. G. Brooks, F. Caruso, S. J. Kent, ACS Nano 2009, 3, 3391; d) B. G. De Geest, M. A. Willart, H. Hammad, B. N. Lambrecht, C. Pollard, P. Bogaert, M. De Filette, X. Saelens, C. Vervaet, J. P. Remon, ACS Nano 2012, 6, 2136; e) B. G. De Geest, M. A. Willart, B. N. Lambrecht, C. Pollard, C. Vervaet, J. P. Remon, J. Grooten, S. De Koker, Angew. Chem. Int. Ed. 2012, 51, 3862. 
[8] a) K. Ariga, A. Vinu, Y. Yamauchi, Q. Ji, J. P. Hill, Bull. Chem. Soc. Jpn. 2012, 85, 1; b) F. Tang, L. Li, D. Chen, Adv. Mater. 2012, 24, 1504.

[9] a) Y. Wang, A. Yu, F. Caruso, Angew. Chem. Int. Ed. 2005, 44, 2888; b) J. Cui, Y. Wang, J. Hao, F. Caruso, Chem. Mater. 2009, 21, 4310; c) Y. Wang, A. D. Price, F. Caruso, J. Mater. Chem. 2009, 19, 6451; d) Y. Wang, F. Caruso, Adv. Mater. 2006, $18,795$.

[10] a) J. Cui, Y. Yan, Y. Wang, F. Caruso, Adv. Funct. Mater. 2012, 22, 4718; b) Y. Wang, V. Bansal, A. N. Zelikin, F. Caruso, Nano Lett. 2008, 8, 1741; c) Y. Wang, Y. Yan, J. Cui, L. Hosta-Rigau, J. K. Heath, E. C. Nice, F. Caruso, Adv. Mater. 2010, 22, 4293; d) J. Tan, Y. Wang, X. Yip, F. Glynn, R. K. Shepherd, F. Caruso, Adv. Mater. 2012, 24, 3362.

[11] X. Zhang, M. Oulad-Abdelghani, A. N. Zelikin, Y. Wang, Y. Haîkel, D. Mainard, J.C. Voegel, F. Caruso, N. Benkirane-Jessel, Biomaterials 2010, 31, 1699.

[12] J.-G. Wang, H.-J. Zhou, P.-C. Sun, D.-T. Ding, T.-H. Chen, Chem. Mater. 2010, 22, 3829.

[13] a) G. T. Zugates, D. G. Anderson, S. R. Little, I. E. B. Lawhorn, R. Langer, J. Am. Chem. Soc. 2006, 128, 12726; b) S. F. Chong, R. Chandrawati, B. Städler, J. Park, J. Cho, Y. Wang, Z. Jia, V. Bulmus, T. P. Davis, A. N. Zelikin, F. Caruso, Small 2009, 5, 2601.

[14] H. Kukula, H. Schlaad, M. Antonietti, S. Förster, J. Am. Chem. Soc. 2002, 124, 1658.

[15] X. Banquy, F. Suarez, A. Argaw, J.-M. Rabanel, P. Grutter, J.-F. Bouchard, P. Hildgen, S. Giasson, Soft Matter 2009, 5, 3984.

[16] Y. Yan, Y. Wang, J. K. Heath, E. C. Nice, F. Caruso, Adv. Mater. 2011, 23, 3916.

[17] P. Attard, J. Phys.: Condens. Matter 2007, 19, 473201.

[18] I. N. Sneddon, Int. J. Eng. Sci. 1965, 3, 47. 


\section{The table of contents:}

\section{Submitted to

\section{Mechanically Tunable, Self-Adjuvanting Nanoengineered Polypeptide Particles}

Jiwei Cui, Robert De Rose, James P. Best, Angus P. R. Johnston, Sheilajen Alcantara, Kang Liang, Georgina K. Such, Stephen J. Kent, * and Frank Caruso*

Keywords: vaccine delivery, nanotechnology, mesoporous silica, polypeptide particles, CpG, adjuvant

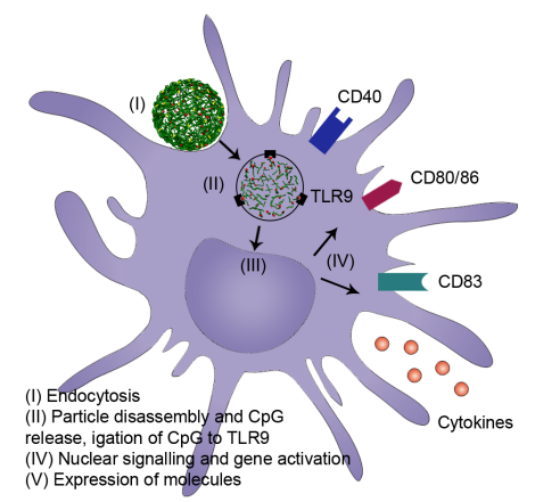

DNA-loaded polypeptide particles are prepared via mesoporous silica templated assembly for the delivery of adjuvants. The elasticity and cargo loading capacity of the obtained particles can be tuned by the amount of cross-linker used to stabilize the polypeptide particles. The use of polypeptide particles as biocarriers provides a promising method for vaccine delivery. 


\section{Submitted to MATERIALS}

Scheme 1. a) Schematic illustration of the preparation of PGA-CpG particles via templating MS particles. (I) Loading PGA-CpG conjugates into MS particles, (II) Cross-linking PGACpG conjugates via thiol-disulfide exchange, (III) removing silica templates to result in PGA-CpG particles. b) Cell association and activation with PGA-CpG particles. (I) Particle endocytosis, (II) dissociation of the particle in an endosome, followed by release of CpG and ligation with TLR9, (III) Nuclear signaling, gene activation and expression of activating molecules, and (IV) expression of activating molecules on the pDC surface, including CD40 and CD83.
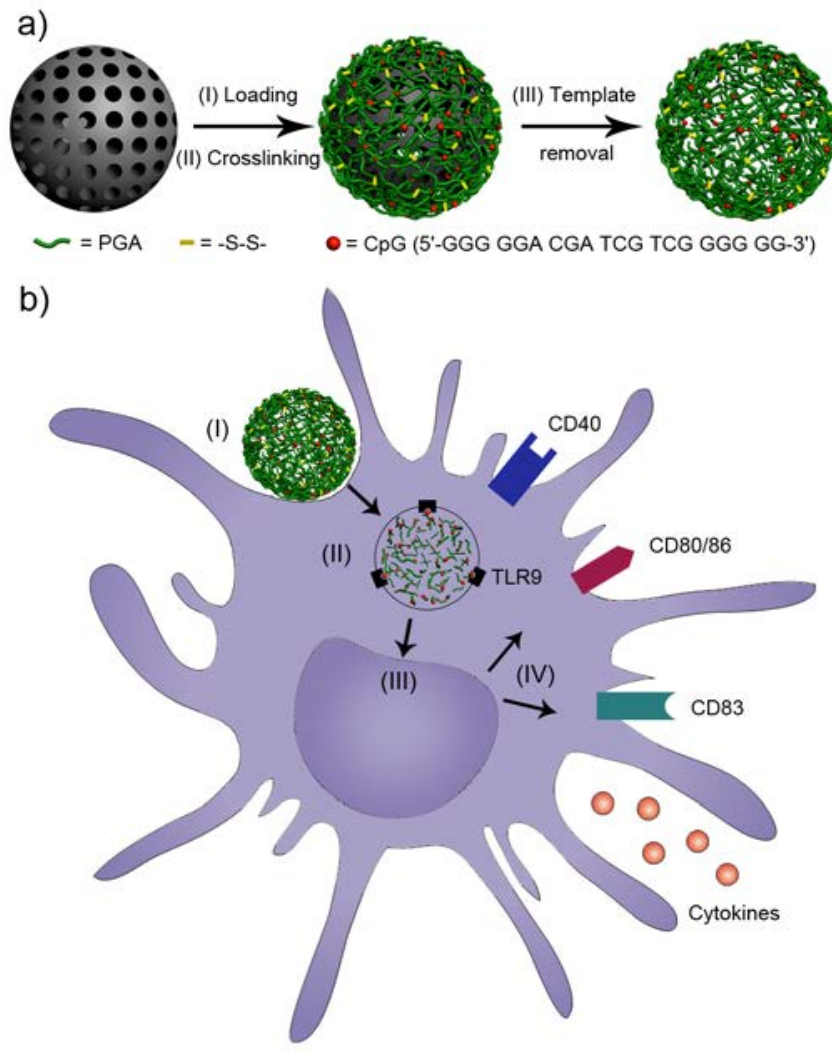


\section{ADVANCED \\ Submitted to MATERIALS}

Figure 1. a) Fluorescence microscopy and b) TEM images of the PGA-CpG particles. 1-6) The cross-linker concentrations were $0.05,0.1,0.2,0.5,1$, and $2 \mathrm{mg} \mathrm{mL}^{-1}$, respectively. The green fluorescence was from FITC-labeled CpG.
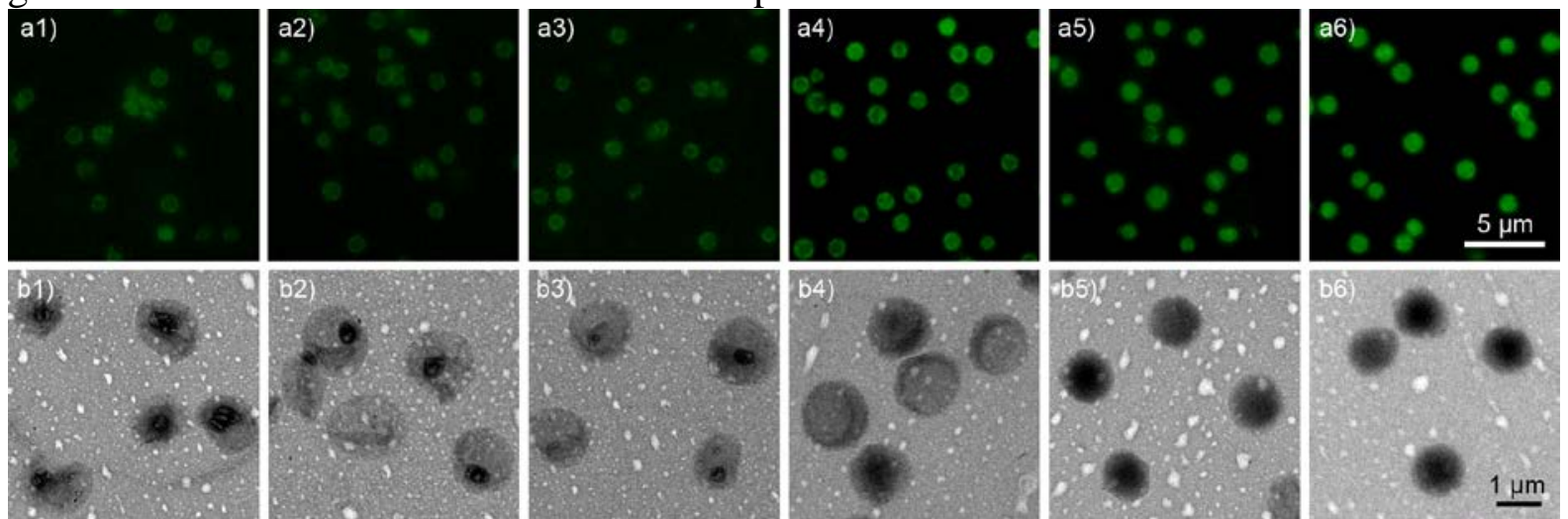


\section{Submitted to

Figure 2. a) Young's modulus of the PGA-CpG particles ( $\boldsymbol{\square}$ ) and $\mathrm{CpG}$ amount per particle $(\mathrm{O})$ as a function of cross-linker concentration. b) Comparison of the Young's modulus of the PGA-control and PGA-CpG particles (cross-linker concentration, $1.0 \mathrm{mg} \mathrm{mL}^{-1}$ ).
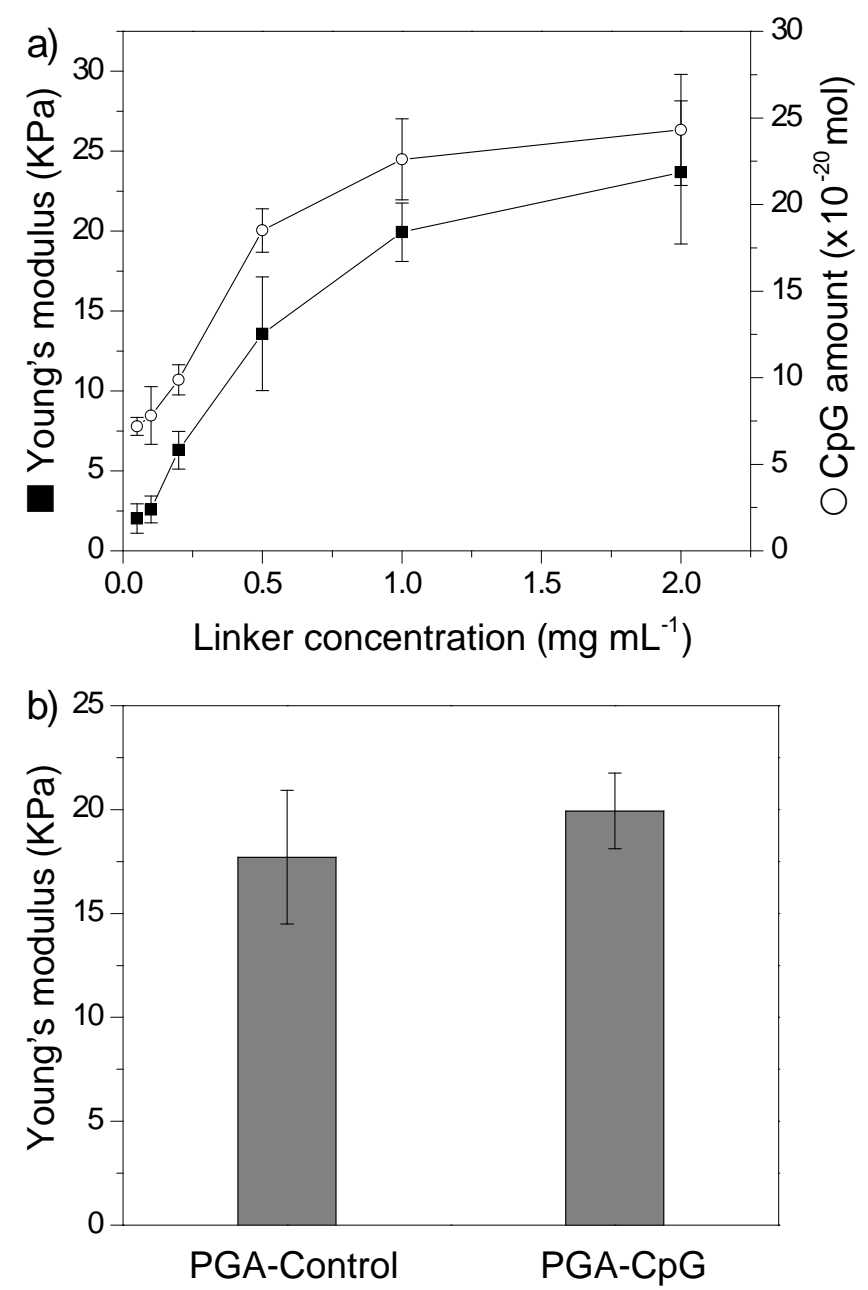
Figure 3. a) pDC association and b) activation (CD83+/CD40+) for the polymer particles as a function of linker concentration.
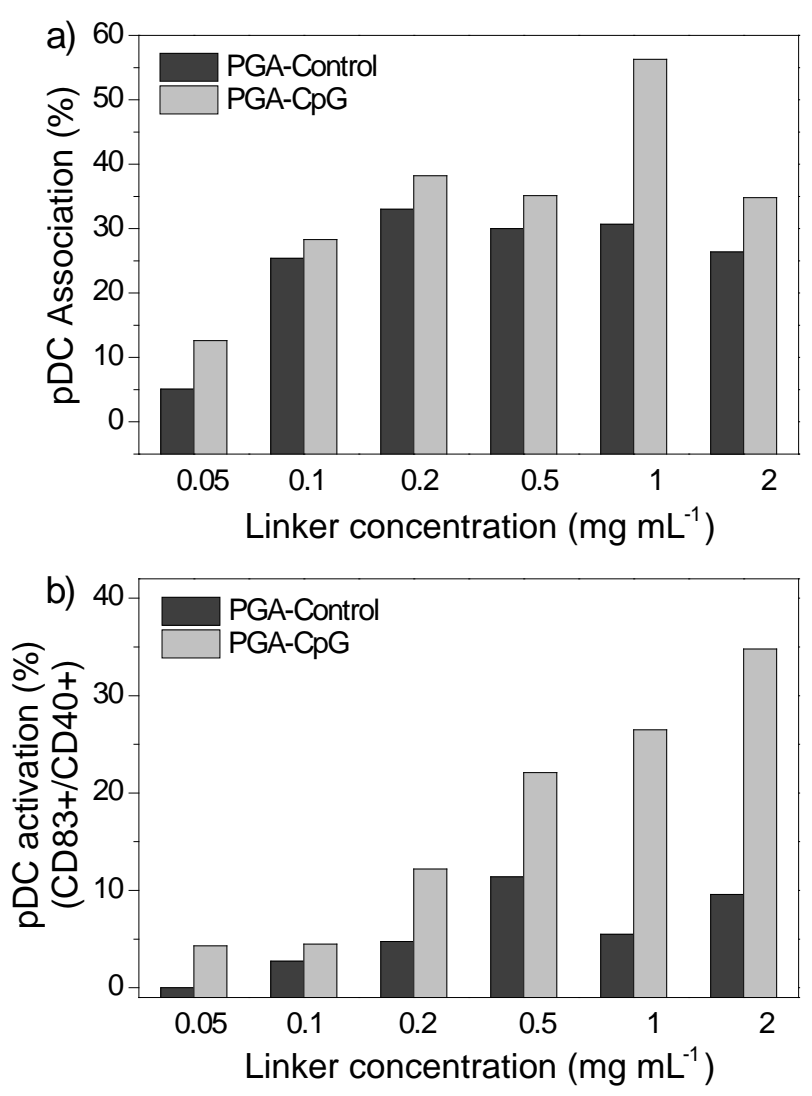


\section{University Library}

\section{- M M N E R VA A gateway to Melbourne's research publications}

Minerva Access is the Institutional Repository of The University of Melbourne

\section{Author/s:}

Cui, J;De Rose, R;Best, JP;Johnston, APR;Alcantara, S;Liang, K;Such, GK;Kent, SJ;Caruso, F

Title:

Mechanically Tunable, Self-Adjuvanting Nanoengineered Polypeptide Particles

Date:

2013-07-05

\section{Citation:}

Cui, J., De Rose, R., Best, J. P., Johnston, A. P. R., Alcantara, S., Liang, K., Such, G. K., Kent, S. J. \& Caruso, F. (2013). Mechanically Tunable, Self-Adjuvanting Nanoengineered Polypeptide Particles. ADVANCED MATERIALS, 25 (25), pp.3468-3472. https:// doi.org/10.1002/adma.201300981.

Persistent Link:

http://hdl.handle.net/11343/123301 